\section{( OPEN ACCESS}

\title{
Results of 2 years of treatment with as-needed ranibizumab reinjection for polypoidal choroidal vasculopathy
}

\author{
Taiichi Hikichi, Makoto Higuchi, Takuro Matsushita, Shoko Kosaka, Reiko Matsushita, \\ Kimitaka Takami, Hideo Ohtsuka, Hirokuni Kitamei, Shoko Shioya
}

Department of Ophthalmology, Ohtsuka Eye Hospital, Sapporo, Japan

\section{Correspondence to} Dr Taiichi Hikichi, Department of Ophthalmology, Ohtsuka Eye Hospital, Kita-16 Nishi-4, Kita-ku, Sapporo 001-0016, Japan; taiichi-hikichi@ hokkaido.med.or.jp

Received 25 September 2012 Revised 14 January 2013 Accepted 21 January 2013 Published Online First 21 February 2013

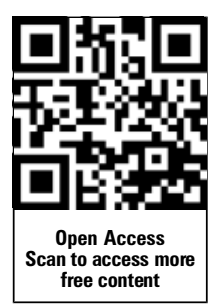

To cite: Hikichi T, Higuchi M, Matsushita T, et al. Br J Ophthalmol 2013;97:617-621.

\begin{abstract}
Purpose To investigate the 2-year outcomes of three monthly intravitreal ranibizumab injections followed by as-needed reinjections to treat polypoidal choroidal vasculopathy (PCV).

Methods Seventy-five consecutive eyes with naïve symptomatic PCV with 2 years of follow-up after treatment were studied prospectively.

Results The mean $( \pm S D)$ numbers of injections were $4.2 \pm 1.3$ that included three monthly injections in the

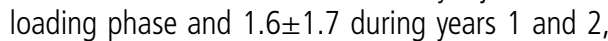
respectively (mean 2-year total, 5.6 \pm 1.9 ). The baseline logarithm of the minimum angle of resolution visual acuity (VA) was $0.59 \pm 0.51$ that improved significantly ( $p=0.001$ for both comparisons) to $0.37 \pm 0.33$ and $0.41 \pm 0.40$ at 1 and 2 years, respectively, after the first injection. Although no significant difference was found between years 1 and 2 after the first injection, the VA tended to decrease slightly during year 2 . The improved foveal thickness was maintained during year 2 . Thirty (40\%) eyes and 19 (25\%) eyes, respectively, at years 1 and 2 after the first injection had no polypoidal lesions on indocyanine green angiography. A branching vascular network (BVN) remained in all eyes 2 years after the first injection and tended to increase in size during year 2 . Conclusions The 2-year outcomes showed significant VA and foveal thickness improvements in eyes with PCV. During year 2, the magnitude of the improvement was lower compared with year 1. An as-needed reinjection schedule might not prevent polypoidal lesions or BVNs from regrowing. Further investigations should establish a treatment strategy for PCV.
\end{abstract}

Polypoidal choroidal vasculopathy (PCV), a distinct clinically relevant exudative macular disorder, is characterised by a network of vessels with two distinct components: a complex of branching vessels and multiple, terminal, reddish-orange polypoidal lesions. ${ }^{1-4}$ Half the number of eyes had a relatively favourable natural outcome, but the other half had persistent leakage or repeated bleeding and poor visual outcomes. ${ }^{3}$

The 1-year results of photodynamic therapy (PDT) for PCV showed that PDT maintained or improved vision. ${ }^{5-12}$ Although in most eyes with PCV, the polypoidal lesions initially resolve after PDT, indocyanine green angiography (ICGA) has shown that the branching vascular networks (BVNs) persist in most eyes. ${ }^{11}$ The long-term visual outcomes after PDT were not good because of the high frequency of recurrent polypoidal lesions and enlargement and neovascular changes involving the BVNs. ${ }^{13-17}$

$\mathrm{We}^{18}$ previously reported that monthly intravitreal injections of ranibizumab for 3 months followed by an as-needed reinjection schedule for eyes with PCV in Japanese patients resulted in continued visual acuity (VA) improvement that was maintained throughout 12 months of follow-up, and the mean VA improved more than 0.2 logarithm of the minimum angle of resolution (logMAR) unit in 81 eyes of 78 patients with PCV. Considering the outcomes of PDT for PCV, since long-term follow-up is essential for evaluation of ranibizumab therapy for PCV, those patients were then followed for 2 years after the first ranibizumab injection. The current study reports the full 2-year results of monthly intravitreal injections of $0.5 \mathrm{mg}$ of ranibizumab for 3 months followed by a reinjection schedule based on need for treating PCV in Japanese patients.

\section{METHODS}

This prospective, consecutive study investigated the 2-year results of one intravitreal $0.5 \mathrm{mg}$ injection of ranibizumab monthly for 3 months followed by an as-needed reinjection schedule to treat PCV. After 13 March 2009, when ranibizumab was approved for use to treat age-related macular degeneration (AMD) in Japan, all patients with AMD, including $\mathrm{PCV}$, were treated with a $0.5 \mathrm{mg}$ intravitreal injection of ranibizumab for 3 months followed by a reinjection schedule based on need at the Ohtsuka Eye Hospital. Eighty-five consecutive eyes of 82 prospective, treatment-naïve Japanese patients with symptomatic PCV received ranibizumab therapy. These patients had intraretinal fluid, subretinal fluid, and pigment epithelial detachment (PED). There were no exclusion criteria regarding the baseline VA or lesion size. Although PCV was diagnosed based on the fundus or ICGA findings or both, and the diagnostic criteria of the Japanese Study Group of PCV, ${ }^{19}$ PCV was diagnosed in all eyes after the presence of polypoidal lesions was confirmed on ICGA. All eyes in the current study were included in our previous study, ${ }^{18}$ which reported the 1-year outcomes of ranibizumab monotherapy for PCV. The current research followed the tenets of the Declaration of Helsinki; all subjects provided informed consent after they received an explanation of the study protocol. The institutional review board at Ohtsuka Eye Hospital prospectively approved the study. 
At the visit at which the first injection of ranibizumab was administered, a complete ophthalmic examination was undertaken including VA measurements with the Landolt ring chart, digital simultaneous fluorescein angiography (FA), ICGA using confocal scanning laser ophthalmoscopy (Heidelberg Retina Angiograph II, Heidelberg Engineering, Dossenheim, Germany), and time-domain optical coherence tomography (OCT) (OCT 3000, Zeiss Humphrey Instruments, Dublin, California, USA). Six radial line scans through the centre of the foveal lesions were used to determine the presence of fluid in the macula. The foveal thickness was determined based on the average foveal thickness on the vertical and horizontal scans. The foveal thickness on the vertical and horizontal scans was measured manually from the inner retinal surface to the retinal pigment epithelium (RPE) line. ${ }^{20}$ The foveal thickness in eyes with a PED was defined as the length of the line from the inner retinal surface to the point at right angles to the line between the edges of the elevated RPE.

Ophthalmic examinations that included measurement of VA and OCT were performed monthly. The best-corrected VA was obtained in an examination room with 200-lux lighting with the patient seated $5 \mathrm{~m}$ from a Landolt's ring chart. When measuring the VA in patients with poor vision, the chart was moved closer to the patients to determine the smallest letter that they could see at a shorter distance. FA and ICGA were performed 3, 6, 12 and 24 months after the first ranibizumab injection. The presence or absence of polypoidal lesions was determined based on the ICGA findings. If no apparent polypoidal lesions were observed, they were considered to have resolved. The planimetric size of the BVNs was calculated by manual measurement using National Institutes of Health Image software. The borders of the BVNs were outlined manually on the ICGA image in the software.

After intravitreal injection of ranibizumab monthly for 3 months, additional injections were administered if any of the following occurred: ${ }^{21} 22$ (1) VA loss of at least $0.1 \operatorname{logMAR}$ unit (equivalent to 5 letters of the Early Treatment Diabetic Retinopathy Study chart) judged from logMAR converted from decimal VA, with evidence of fluid at the macula on OCT images. If necessary, the chart was moved closer to the patients to determine whether the converted $\log$ MAR unit changed at least $0.1 ;(2)$ any qualitative change in the appearance of the OCT images that suggested recurrent fluid in the macula including enlargement of a PED or (3) persistent fluid excluding persistent PED on OCT 1 month after the previous injection. All criteria were based on comparisons with the previous month's examination. If any criterion for reinjection was fulfilled, the intravitreal injection was administered. One clinician (TH) examined all patients and determined the need for reinjection during the 2-year follow-up period.

The VA results were converted to $\log$ MAR for analysis. Statistical analysis was performed using the SPSS V.11.5.1 for Windows software package (SPSS, Chicago, Illinois, USA).

\section{RESULTS}

Of 85 consecutive eyes with PCV, 4 (5\%) eyes were lost to follow-up during the first year as reported previously. ${ }^{18}$ In another six eyes of five patients, the follow-up examinations were interrupted during the second year, the reasons for interruption of the examination being poor physical condition in three eyes of two patients and stable fundus findings and VA in three eyes. Thus, 75 eyes of 73 patients $(51$ men, 22 women) were followed for more than 2 years after the first injection, and analysed (table 1$)$. The mean $( \pm S D)$ numbers of reinjections were $1.2 \pm 1.3$ during the first year and 1.6 \pm 1.7 during the
Table 1 Baseline characteristics of 75 eyes of 73 patients with polypoidal choroidal vasculopathy

\begin{tabular}{ll}
\hline Age, years $($ mean \pm SD) & $71 \pm 9$ \\
Men (eyes, \%) & 51 patients $(52,69)$ \\
LogMAR VA (mean \pm SD) & $0.59 \pm 0.51$ \\
Foveal thickness, $\mu \mathrm{m}(\text { mean } \pm S D)^{2}$ & $324 \pm 80$ \\
Planimetric size of BVN $\left(\mathrm{mm}^{2}\right)$ & $3.38 \pm 1.14$ \\
Baseline cluster of grape-like polypoidal lesions $(\%)$ & 10 eyes $(13)$ \\
Subretinal haemorrhage $\geq 1$ DD $(\%)$ & 24 eyes $(32)$ \\
PED $\geq 1$ DD $(\%)$ & 19 eyes $(25)$ \\
\hline
\end{tabular}

BVN, branching vascular network; DD, disc diameter; MAR, minimum angle of resolution; PED, pigment epithelial detachment; VA, visual acuity.

second year. The mean $( \pm S D)$ total number of injections, including three monthly injections (the loading phase), during

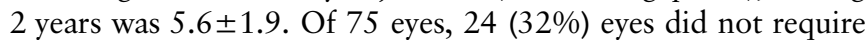
additional injections during the 2nd year, whereas 17 (23\%) eyes required 1, $19(25 \%)$ eyes required 2, $4(5 \%)$ eyes required $3,6(8 \%)$ eyes required $4,1(1 \%)$ eye required 5 , and $4(5 \%)$ eyes required 6 during the 2 nd year. During the 2 -year follow-up period, the total numbers of ranibizumab injections were three in 11 (15\%) eyes, four in 11 (15\%) eyes, five in 17 (23\%) eyes, six in $13(17 \%)$ eyes, seven in $10(13 \%)$ eyes, eight in $4(5 \%)$ eyes, and nine in $9(12 \%)$ eyes. No significant correlation was found between the numbers of injections administered at year 1 and year $2(\mathrm{r}=0.09, \mathrm{p}=0.53$, Pearson's correlation analysis).

Figure 1 shows the changes in the mean $( \pm S D) \operatorname{logMAR} V A$ during the 2-year follow-up period after the first ranibizumab injection. The baseline $\log$ MAR VA was $0.59 \pm 0.51$, which significantly improved 1 month after the third intravitreal injection $(0.40 \pm 0.35)$, and the improved VA was maintained thereafter $(0.37 \pm 0.331$ year after the first injection). The VAs 1 month after the third injection, and 1 year after the first injection were significantly $(p=0.001$, for both comparisons by the Student $t$ test) better than the baseline VA. Although the significant improvement in the VA 2 years after the first injection $(0.41$ $\pm 0.40, p=0.001$, Student $t$ test) was maintained compared with the baseline VA, the VA tended to decrease slightly during the

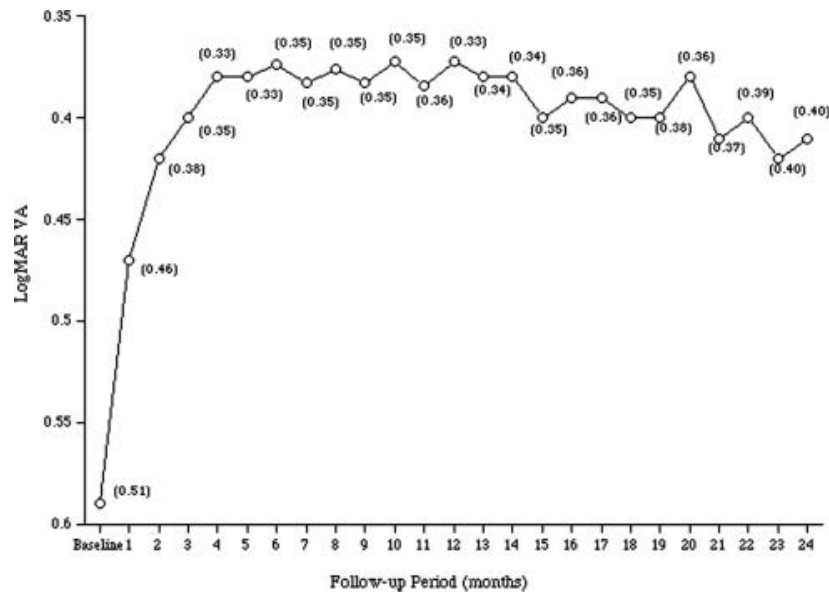

Figure 1 The changes in the mean logarithm of the minimum angle of resolution visual acuity during the 2-year follow-up period after the first ranibizumab injection to treat polypoidal choroidal vasculopathy in Japanese patients. The numbers in the parentheses indicate 1 SD of the mean. 
second year. However, no significant $(p=0.37$, Student $t$ test) difference was found in the VAs between years 1 and 2 after the first injection.

Figure 2 shows the changes in the mean $( \pm S D)$ foveal thickness for the 2-year follow-up period after the first injection. The mean foveal thickness 1 month after the third intravitreal injection $(203 \pm 37 \mu \mathrm{m})$ decreased significantly compared with the mean foveal thickness at baseline $(324 \pm 80 \mu \mathrm{m}, \mathrm{p}=0.001$, Student $t$ test) and was maintained thereafter $(211 \pm 45 \mu \mathrm{m}$ at 1 year after the first injection, $\mathrm{p}=0.001$, Student $\mathrm{t}$ test). During the second year, the improvement in the foveal thickness was maintained, and the mean foveal thickness 2 years after the first injection $(213 \pm 42, p=0.001$, Student $t$ test $)$ improved significantly compared with the baseline foveal thickness.

Of the 75 eyes, the logMAR VA 2 years after the first injection improved or decreased $0.3 \log$ MAR unit or more from the baseline logMAR VA in 25 (33\%) eyes or 7 (10\%) eyes, respectively. The remaining $43(57 \%)$ eyes had less than a 0.3 unit change in the $\log$ MAR VA. The percentage of eyes with improved VA decreased slightly, and the percentage of eyes with decreased VA increased slightly at 2 years after the first injection compared with $(37 \%, 28$ eyes; 7\%, five eyes, respectively) 1 year after the first injection. Of the seven eyes with decreased VA, four eyes had already had a decrease in VA 1 year after the first injection; in the remaining three eyes, a retinal and subretinal haemorrhage that included the fovea developed during the second year, which was thought to cause a serious decrease in the VA. A retinal and subretinal haemorrhage not associated with deterioration of visual function was found in three eyes during the second year. The numbers (mean \pm SD) of injections during the 2-year follow-up period after the first ranibizumab injection were 5.2 \pm 1.8 in eyes with improved VA, 5.8 \pm 2.2 in eyes with decreased VA, and 5.8 \pm 2.1 in eyes with stable VA. These values did not differ significantly $(p>0.600$, for all comparisons by Fisher's protected least significant difference test) among the three groups.

The baseline ICGA showed polypoidal lesions and BVNs in all except four eyes in which a BVN was not detected because of retinal and subretinal haemorrhages. No polypoidal lesions were detected on ICGA in 30 (40\%) eyes 1 year after the first injection, whereas the percentage, which was not significantly different $\left(p=0.056, \chi^{2}\right.$ test), decreased to $25 \%$ (19 eyes) on ICGA 2 years after the first injection. A BVN remained in all

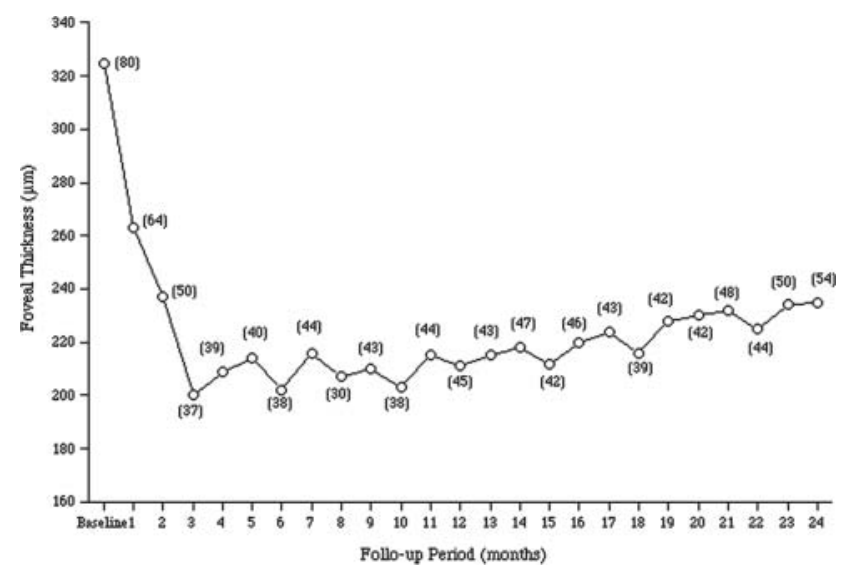

Figure 2 The changes in the mean foveal thickness during the 2-year follow-up period after the first ranibizumab injection to treat polypoidal choroidal vasculopathy in Japanese patients. The numbers in the parentheses indicate 1 SD of the mean. eyes 2 years after the first injection. Of the 71 eyes in which a BVN was detected at baseline, the planimetric size of the BVN 2 years after the first injection increased more than $20 \%$ compared with baseline in $14(20 \%)$ eyes, and decreased more than $20 \%$ compared with baseline in one (1\%) eye (figure 3). Changes in the planimetric size of the BVNs that were less than $20 \%$ occurred in $56(79 \%)$ eyes. Those percentages 1 year after the first injection were 13\% (9 eyes), 4\% (3 eyes), and 84\% (59 eyes), respectively. The percentages in years 1 and 2 did not differ significantly after the first injection $\left(p=0.330, \chi^{2}\right.$ test), but the planimetric size of the BVNs tended to increase.

At baseline, 16 (21\%) of 75 eyes were pseudophakic. No eyes underwent cataract surgery during the first year. Two eyes required surgery during the second year, but the decimal VA was unchanged in the eyes. There were no apparent ocular adverse events attributable to the ranibizumab injections. No episodes of endophthalmitis, uveitis, lens damage, or prolonged intraocular pressure elevation occurred.

\section{DISCUSSION}

Our results showed that three monthly intravitreal injections of ranibizumab followed by an as-needed reinjection schedule improved VA and reduced the amount of macular fluid in patients with PCV, and maintained the improved VA and macular findings throughout 2 years.

However, the following outcomes differed between 1 year and 2 years after the first ranibizumab injection. The first finding was that the increase in the mean logMAR VA from baseline decreased by 0.042 years after the first injection $(0.18)$ compared with 1 year after the first injection $(0.22)$. The percentage of eyes with improved VA of 0.3 or more logMAR unit decreased slightly 2 years after the first injection compared with 1 year after the first injection. The Comparison of Age-Related Macular Degeneration Treatment Trials (CATT) study, ${ }^{23}$ a randomised clinical trial that compared the AMD treatment among four groups by drug (ranibizumab or bevacizumab (Avastin, Genentech, south San Francisco, California, USA)) and dosing regimen (monthly or as-needed treatment), also found that the VA outcomes tended to be slightly worse 2 years after the first injection compared with 1 year after the first injection. As-needed treatment immediately addresses recurrent fluid and may minimise the deterioration of visual function, thus preventing a decline in the improved VA but not eliminating it completely. This seems to be a limitation of an as-needed reinjection schedule. In this study, a retinal and subretinal haemorrhage developed during the second year caused a serious decrease in the VA. Ranibizumab therapy continued even after development of the haemorrhage in those patients. Although deterioration of the RPE was associated with decrease in the VA during the second year in some patients as previously reported, ${ }^{24}$ the decreased $\log$ MAR units were less than 0.3 .

The second finding was worsening of the PCV components, ie, an increased percentage of eyes with polypoidal lesions on ICGA and increased size of the BVN during the second year. In the as-needed treatment group of the CATT study, ${ }^{23}$ a tendency for lesion growth was found during the second year. In as-needed treatment, lesion growth might occur without being detected on OCT, which might result in no retreatment in some eyes. To avoid missing silent lesion growth, ie, the undetectable changes on OCT, angiography occasionally might be required to evaluate lesions. In a study of the long-term efficacy of intravitreal bevacizumab for recurrent leakage due to residual BVNs in PCV after PDT, Wakabayashi and associates ${ }^{25}$ reported that the BVNs enlarged in $44 \%$ eyes despite repeated injections, and 
A

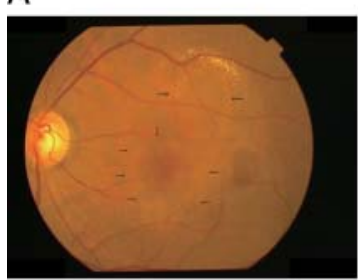

D

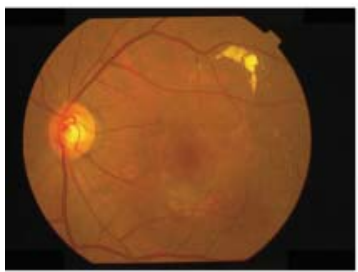

G

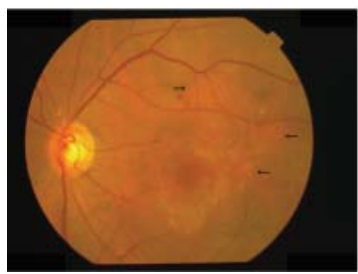

B

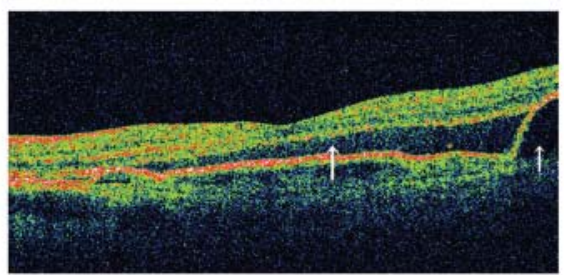

E

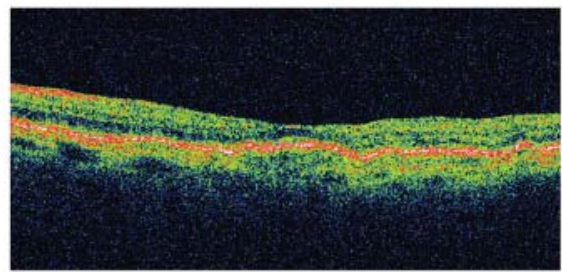

$\mathrm{H}$

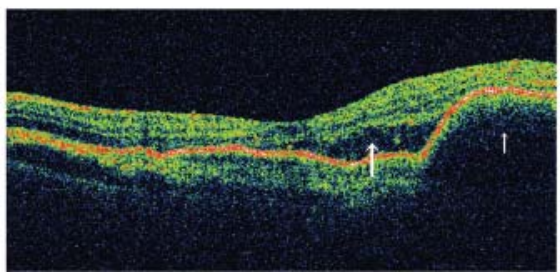

C

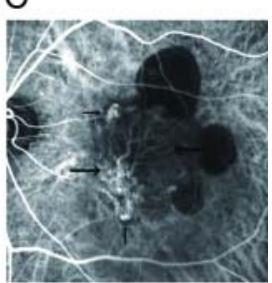

$\mathrm{F}$

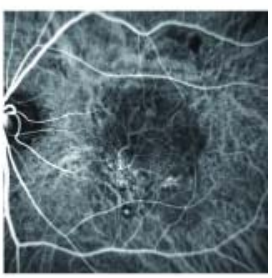

I

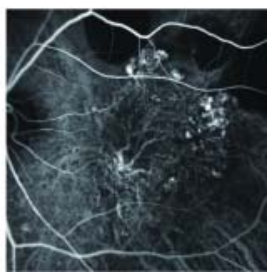

Figure 3 The left eye of a 71-year-old man with polypoidal choroidal vasculopathy. (A) At baseline, a fundus photograph shows a serous retinal detachment (SRD) involving the fovea (small arrows) and a serous pigment epithelial detachment (PED) (large arrows). (B) A horizontal optical coherence tomography (OCT) image shows a subfoveal SRD (large arrow) and PED temporal to the fovea (small arrow). (C) indocyanine green angiography (ICGA) shows polypoidal lesions (small arrows) and a branching vascular network (BVN) (large arrows). The planimetric size of the BVN is $4.33 \mathrm{~mm}^{2}$. (D and E) One year after the first injection of ranibizumab, a fundus photograph and OCT shows resolution of the SRD and PED. (F) ICGA shows regression of polypoidal lesions and no increase in the size of the BVN $\left(4.42 \mathrm{~mm}^{2}\right)$. (G) Two years after the first injection, a fundus photograph shows some elevated orange-red lesions (arrows). (H) A horizontal OCT image shows a recurrent subfoveal SRD (large arrow) and a PED temporal to the fovea (small arrow). (I) ICGA shows progression of the polypoidal lesions and the size of the BVN $\left(5.75 \mathrm{~mm}^{2}\right)$.

explained that this expansion might lead to more mature and less vascular endothelial growth factor-dependent vessels with increased treatment resistance.

Polypoidal lesions might be associated with exudative macular changes. $^{6-10} 13$ In the current study, polypoidal lesions were not detected on ICGA in $30(40 \%)$ eyes 1 year after the first injection; however, that percentage decreased to $25 \%$ (19/75) 2 years after the first injection. The presence of polypoidal lesions might be associated with risk of development of retinal and subretinal haemorrhages. ${ }^{3}$ During the second year of the current study, haemorrhagic events developed in six eyes, three of the six eyes had a $0.3 \log$ MAR unit or greater decrease in $\mathrm{VA}$, and the haemorrhages involved the fovea and were thought to cause a serious decrease in VA.

The limitations of the current study were the lack of a control group, and that the Early Treatment of Diabetic Retinopathy Study chart was not used to measure the VA, and the eyes underwent time-domain OCT, which is inferior to spectraldomain OCT in resolution of images, ${ }^{26}$ during the follow-up period. The strengths included the prospective design, longer follow-up, and a relatively high rate of adherence to the protocol. We speculated that an as-needed reinjection schedule might be limited in its ability to prevent regrowth of polypoidal lesions and BVNs. Since the long-term visual outcomes of PDT were not good owing to the high frequency of recurrent polypoidal lesions resulting from residual BVNs, ${ }^{13-17}$ the limitations of an as-needed reinjection schedule require further observations, and a future study comparing as-needed therapy to a more frequent regimen, such as an injection every two or three months, or treat and extend is warranted.

Contributors $\mathrm{TH}$ : conception, design, acquisition of data, analysis, drafting and revising the article, final approval of all the versions. MH, TM, SK, RM, KT and HO: conception, design and final approval of all the versions. HK: interpretation of data and final approval of all the versions. SS: interpretation of data and final approval of all the versions.

Funding Dr Hikichi received lecture fees from Novartis Pharma Japan, Bayer. Japan, Santen and Alcon Japan.

\section{Competing interests None.}

Ethics approval The institutional review board at Ohtsuka Eye Hospital prospectively approved the study.

Provenance and peer review Not commissioned; externally peer reviewed.

Open Access This is an Open Access article distributed in accordance with the Creative Commons Attribution Non Commercial (CC BY-NC 3.0) license, which permits others to distribute, remix, adapt, build upon this work non-commercially, and license their derivative works on different terms, provided the original work is properly cited and the use is non-commercial. See: http://creativecommons.org/ licenses/by-nc/3.0/

\section{REFERENCES}

1 Yannuzzi LA, Sorenson J, Spaide RF, et al. Idiopathic polypoidal choroidal vasculopathy (IPCV). Retina 1990;10:1-8.

2 Spaide RF, Yannuzzi LA, Slakter JS, et al. Indocyanine green videoangiography of idiopathic polypoidal choroidal vasculopathy. Retina 1995;15:100-10

3 Uyama M, Wada M, Nagai Y, et al. Polypoidal choroidal vasculopathy: natural history. Am J Ophthalmol 2002;133:639-48.

4 Imamura Y, Engelbert M, lida T, et al. Polypoidal choroidal vasculopathy: a review. Surv Ophthalmol 2010;55:501-15. 
5 Chan WM, Lam DS, Lai TYY, et al. Photodynamic therapy with verteporfin for symptomatic polypoidal choroidal vasculopathy. One-year results of a prospective case series. Ophthalmology 2004;111:1576-84.

6 Saito M, lida T, Nagayama D. Photodynamic therapy with verteporfin for age-related macular degeneration or polypoidal choroidal vasculopathy: comparison of the presence of serous retinal pigment epithelial detachment. $\mathrm{Br} J$ Ophthalmol 2008:92:1642-7.

7 Spaide RF, Donsoff I, Lam DL, et al. Treatment of polypoidal choroidal vasculopathy with photodynamic therapy. Retina 2002;22:529-35.

8 Quaranta M, Maget-Faysse M, Coscas G. Exudative idiopathic polypoidal choroidal vasculopathy and photodynamic therapy with verteporfin. Am J Ophthalmol 2002;134:277-80.

9 Eandi $\mathrm{CM}$, Ober MD, Freund $\mathrm{KB}$, et al. Selective photodynamic therapy for neovascular age-related macular degeneration with polypoidal choroidal neovascularization. Retina 2007;27:825-31.

10 Gomi F, Ohji M, Sayanagi K, et al. One-year outcomes of photodynamic therapy in age-related macular degeneration and polypoidal choroidal vasculopathy in Japanese patients. Ophthalmology 2008;115:141-6.

11 Wakabayashi T, Gomi F, Sawa M, et al. Marked vascular changes of polypoidal choroidal vasculopathy after photodynamic therapy. Br J Ophthalmol 2008;92:936-40.

12 Hikichi T, Ohtsuka H, Higuchi M, et al. Factors predictive of visual acuity outcomes 1 year after photodynamic therapy in Japanese patients with polypoidal choroidal vasculopathy. Retina 2011;31:857-65.

13 Akaza E, Mori R, Yuzawa M. Long-term results of photodynamic therapy of polypoidal choroidal vasculopathy. Retina 2008;28:717-22.

14 Kurashige Y, Otani A, Sasahara M, et al. Two-year results of photodynamic therapy for polypoidal choroidal vasculopathy. Am J Ophthalmol 2008;146:513-19.

15 Tsuchiya D, Yamamoto T, Kawasaki R, et al. Two-year visual outcomes after photodynamic therapy in age-related macular degeneration patients with or without polypoidal choroidal vasculopathy lesions. Retina 2009;29:960-5.

16 Akaza E, Yuzawa M, Mori R. Three-year follow-up results of photodynamic therapy for polypoidal choroidal vasculopathy. Jpn J Ophthalmol 2011:55:39-44.
17 Sayanagi K, Gomi F, Sawa M, et al. Long-term follow-up of polypoidal choroidal vasculopathy after photodynamic therapy with verteporfin. Graefes Arch Clin Exp Ophthalmol 2007;245:1569-71.

18 Hikichi T, Higuchi M, Matsushita T, et al. One year results of three monthly ranibizumab injections and as-needed reinjections for polypoidal choroidal vasculopathy in Japanese patients. Am J Ophthalmol 2012;154:117-24.

19 Japanese Study Group of Polypoidal Choroidal Vasculopathy. Criteria for diagnosis of polypoidal choroidal vasculopathy [in Japanese]. Nippon Ganka Gakkai Zasshi 2005; 109:417-27.

20 Costa RA, Calucci D, Skaf M, et al. Optical coherence tomography 3: automatic delineation of the outer neural retinal boundary and its influence on retinal thickness measurements. Invest Ophthalmol Vis Sci 2004;45:2399-406.

21 Fung AE, Lalwani GA, Rosenfeld PJ, et al. An optical coherence tomographyguided, variable dosing regimen with intravitreal ranibizumab (Lucentis) for neovascular age-related macular degeneration. Am J Ophthalmol 2007; 143:566-83.

22 Lalwani GA, Rosenfeld PJ, Fung AE, et al. A variable-dosing regimen with intravitreal ranibizumab for neovascular age-related macular degeneration: year 2 of the PrONTO study. Am J Ophthalmol 2009;148:43-58.

23 Comparison of Age-related Macular Degeneration Treatments Trials (CATT) Research Group Writing Committee. Ranibizumab and bevacizumab for treatment of neovascular age-related macular degeneration. Two-year results. Ophthalmology 2012:119:1388-98

24 Rosenfeld PJ, Shapiro H, Tuomi L, et al. Characteristics of patients losing vision after 2 years of monthly dosing in the phase III ranibizumab clinical trials. Ophthalmology 2011;118:523-30.

25 Wakabayashi T, Gomi F, Sawa M, et al. Intravitreal bevacizumab for exudative branching vascular networks in polypoidal choroidal vasculopathy. $\mathrm{Br} J$ Ophthalmol 2012:96:394-9.

26 Iwama D, Hangai M, Ooto $S$, et al. Automated assessment of drusen using three-dimensional spectral-domain optical coherence tomography. Invest Ophthalmol Vis Sci 2012;53:1576-83. 